\title{
Measurement and Minimisation of the Mapping Entropy of a Coarse-Grained Biomolecular System ${ }^{+}$
}

\section{Marco Giulini}

University of Trento, Trento, Italy

+ Presented at the Entropy 2021: The Scientific Tool of the 21st Century, 5-7 May 2021; Available online: https://sciforum.net/conference/Entropy2021/.

Published: 5 May 2021

All-atom Molecular Dynamics (MD) is the standard approach to perform in silico simulations of biomolecular systems. Despite its central role in modern computational biophysics, MD cannot span the time scales where the majority of relevant biological processes take place. An alternative is represented by coarse-grained (CG) modelling [1], that is, those lower-resolution representations of the system which aim at effectively reducing the number of degrees of freedom of a biomolecule in order to reach previously inaccessible time scales. Among the several statistical mechanics-based CG'ing techniques, we focused on those that measure the difference in information content between the coarsegrained and the all-atom system. We developed a protocol [2] able to compute the Mapping Entropy, which quantifies the amount of information retained upon the process of CG'ing due only to the choice of the Mapping. Our approach can therefore provide the user with the subset of sites which are maximally informative about the original, fully atomistic system. Tests conducted over a set of wellknown proteins showed that regions retained with high probability are often related to the biological function of the molecule.

\section{References}

1. Noid, W.G. Perspective: Coarse-grained models for biomolecular systems. J. Chem. Phys. 2013, 139, 09B201_1.

2. Giulini, M.; Menichetti, R.; Shell, M.S.; Potestio, R. An Information-Theory-Based Approach for Optimal Model Reduction of Biomolecules. J. Chem. Theory Comput. 2020, 16, 6795-6813.

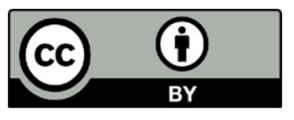

(C) 2021 by the author. Licensee MDPI, Basel, Switzerland. This article is an open access article distributed under the terms and conditions of the Creative Commons Attribution (CC BY) license (http://creativecommons.org/licenses/by/4.0/). 